\title{
UTILIZAÇÃO DE ÁLCOOL ABSOLUTO NO TRATAMENTO DAS MALFORMAÇÕES VENOSAS*
}

\author{
Marco Antonio Lourenço ${ }^{1}$, Claudia Stein Gomes ${ }^{1}$, Cristina Veronese Beffa ${ }^{2}$, \\ Fernando Silveira Picheth ${ }^{3}$
}

Resumo 0 tratamento das malformações venosas pode ser realizado por ressecção cirúrgica ou esclerose farmacológica. Este estudo mostra os resultados de 12 pacientes com angiodisplasia submetidos a múltiplas aplicações diretas de etanol na lesão, após exame clínico e angiografia venosa percutânea. Dez pacientes referiram melhora dos sintomas e diminuição do tamanho da lesão. Todos os pacientes queixaram-se de ardência durante a aplicação, que cessou no máximo em 24 horas. As complicações encontradas foram três casos de úlceras pequenas na pele e um caso de hiperemia no membro tratado, associada a linfonodomegalia dolorosa. Concluiu-se que o tratamento escleroterapêutico das malformações venosas com infusão de pequenos volumes de etanol apresenta as vantagens de ser um tratamento seguro, eficaz, simples, fácil e rápido, de baixo custo, bem tolerado e com poucas complicações.

Unitermos: Etanol. Malformações vasculares venosas. Angiodisplasias. Angiografia.

Abstract Absolute alcohol for the treatment of venous malformations.

Venous malformations can be treated by surgical resection or pharmacological sclerotherapy. This study shows the results of 12 patients with angiodysplasia whose lesions were injected several times with ethanol, after clinical examination and percutaneous venous angiography. Ten patients presented improvement of symptoms and reduction of the size of the lesion. All patients complained about a burning sensation during the procedure that disappeared after 24 hours. Three patients presented small skin ulcers and one had erythema and painful enlarged lymph nodes in the treated limb. We concluded that sclerotherapy with infusion of small volumes of ethanol for the treatment of venous malformations is an effective, safe, simple, easy and quick treatment, that has few complications, low cost, and is well tolerated.

Key words: Ethanol. Venous malformations. Angiodisplasy. Angiography.

\section{INTRODUÇÃO}

A malformação vascular é uma entidade conhecida há mais de 100 anos, porém sua classificação sempre foi um tanto complexa. Vários autores estudaram e classificaram as angiodisplasias, de acordo com seus aspectos morfológicos, muitas vezes dando origem a várias denominações para um mesmo tipo morfológico da lesão. Somente em 1982, Mulliken e Glowacki introduziram uma classificação mais moderna e simplificada, baseada na característica endote-

* Trabalho realizado no Serviço de Angiologia e Cirurgia Vascular da Irmandade da Santa Casa de Misericórdia de Curitiba - Pontifícia Universidade Católica do Paraná, Curitiba, PR.

1. Médicos Cirurgiões do Serviço de Angiologia e Cirurgia Vascular da Irmandade da Santa Casa de Misericórdia de Curitiba - Pontifícia Universidade Católica do Paraná.

2. Médica Residente do Serviço de Angiologia e Cirurgia Vascular da Irmandade da Santa Casa de Misericórdia de Curitiba - Pontifícia Universidade Católica do Paraná.

3. Chefe do Serviço de Cirurgia Vascular da Irmandade da Santa Casa de Misericórdia de Curitiba - Pontifícia Universidade Católica do Paraná.

Endereço para correspondência: Dr. Marco Antonio Lourenço. Avenida Silva Jardim, 1364, apto. 1204, Rebouças. Curitiba, PR, 80250-200. E-mail: malourenço@onda.com.br

Aceito para publicação em 24/7/2000. lial do vaso, contendo apenas duas categorias principais: hemangiomas e malformações vasculares propriamente di$\operatorname{tas}^{(1-4)}$

Os hemangiomas usualmente não estão presentes ao nascimento, aparecem no primeiro mês de vida, e em $95 \%$ dos casos sofrem um processo de regressão que pode ser rápido ou prolongar-se até os dez anos de idade. Histologicamente, são caracterizados por hiperplasia endotelial na fase proliferativa, e fibrose, infiltração gordurosa e celularidade diminuída na fase involutiva. Nas malformações, as lesões já estão presentes ao nascimento, crescem proporcionalmente ao desenvolvimento do organismo e não regridem espontaneamente. São coleções de vasos anormais com endotélio preservado, podendo ser subdivididas em arteriais, venosas, linfáticas, ou uma combinação destas ${ }^{(\mathbf{1 , 5}, \mathbf{6})}$.

Para selecionar o tratamento mais adequado, Jackson et al., em 1993, classificaram as malformações vasculares, segundo a dinâmica circulatória, em lesões de baixo fluxo ou hemodinamica- mente inativas (malformações venosas e capilares) e lesões de alto fluxo ou ativas (comunicações arteriovenosas) ${ }^{(7)}$.

As manifestações clínicas mais freqüentes incluem transtornos estéticos, dor e limitação funcional da área anatômica que se assenta à malformação vascular, particularmente após exercícios ou em dias quentes ${ }^{(\mathbf{1 , 5}, \mathbf{8 , 9})}$.

A anamnese e o exame físico são suficientes, na maioria das vezes, para o diagnóstico das malformações vasculares; entretanto, a utilização de exames complementares, como raios- $\mathrm{X}$ simples, Doppler ultra-som, tomografia computadorizada, ressonância magnética ou angiografia por punção direta, possibilitam identificar a extensão da lesão, permitindo um melhor planejamento terapêutico ${ }^{(10-12)}$.

A angiografia percutânea possibilita a visibilização de "lagos venosos" e eventual opacificação de suas veias de drenagem para o sistema venoso profundo, auxiliando no diagnóstico e planejamento terapêutico. O tratamento das malformações venosas tem sua indica- 
ção estética ou funcional, podendo ser realizado por meio de ressecção cirúrgica ou esclerose farmacológica. As lesões que crescem envolvendo músculos, nervos e vasos sanguíneos dificultam as delimitações com os tecidos normais; muitas vezes é impossível a ressecção do tumor sem extirpação da massa muscular, e com freqüência ocorrem grandes perdas sanguíneas, de difícil controle. Em alguns casos pode ser feita a esclerose farmacológica e, após fibrose do tumor, sua ressecção ${ }^{(13)}$.

$\mathrm{Na}$ emboloterapia podem ser usados diversos materiais, como álcool absoluto ou etanol ${ }^{(1,3,4,7,13-20)}$, Ethibloc ${ }^{\circledast}$ (Ethnor, Ethicon $)^{(1,15,21)}$, micropartículas de polivinil-álcool ${ }^{(1,20)}$, Gelfoam ${ }^{\circledR}$ (Spongostam film, Ferrosan, Söborg, Dinamarca ${ }^{(1,19)}$, colas cianocrilatos - Hystoacril ${ }^{\circledR}$ (Braun, Melsungen, Alemanha) ${ }^{(1)}$, espirais de Gianturco $^{\otimes}$ (Cook Co., Bjaeverskov, Dinamarca $)^{(1)}$, ou associação destes.

O etanol é um agente esclerosante efetivo e causa necrose da parede dos vasos, com rompimento imediato de células vermelhas e subseqüente trombose, levando, posteriormente, à formação de fibrose, que regride gradualmente o tamanho da malformação ${ }^{(4)}$.

\section{MATERIAL E MÉTODOS}

No período de julho/1997 a dezembro/1999, foram avaliados 12 pacientes com malformações vasculares puramente venosas, de baixo fluxo, em extremidades, provenientes do Ambulatório de Cirurgia Vascular da Santa Casa de Misericórdia de Curitiba. Sete pacientes tinham as lesões em membros superiores, e cinco, em membros inferiores. A idade variou entre 17 e 65 anos (média de 27,6 anos), sendo nove pacientes do sexo feminino e três do sexo masculino. A localização das lesões, sinais e sintomas, tratamentos prévios e o diagnóstico são mostrados na Tabela 1. Oito pacientes tinham suas anomalias desde o nascimento, e em quatro a malformação foi percebida, em um deles, aos 11 anos de idade, em outro aos 13, em outro aos 15 , e em outro aos 16 anos de idade. Apenas dois pacientes tinham sido submetidos a tratamento cirúrgico prévio, num total de três ressecções sem sucesso.

Após anamnese e exame clínico minucioso, todos os pacientes foram submetidos a angiografia venosa percutânea para avaliar a extensão da lesão, presença de veias de drenagem para sistema venoso profundo e planejar o tratamento. Excluíram-se os pacientes com sinais angiográficos de acometimento do sistema venoso profundo, mesmo com o uso de garrote, e os casos em que se evidenciou, clinicamente, a existência de comunicações arteriovenosas.

A angiografia foi realizada em aparelho Angioskop-D ${ }^{\circledR}$ (Siemens, Elema) com subtração digital, após punção direta do local a ser examinado, injetando-se lenta e manualmente solução de contraste Reliev $^{\circledR}$ 60\% (Berlimed, Madrid, Espanha), observando-se opacificação dos espaços venosos, contornos e sistemas de drenagem, conforme mostram as Figuras $1 \mathrm{e} 2$.

Para a esclerose percutânea procediase a assepsia local e um "scalp" 21 era introduzido perpendicularmente à pele, até ser alcançado um "lago venoso", o que era verificado por um bom refluxo de sangue, conforme se vê na Figura 3. Utilizou-se garrote proximal ao local da punção, nos pacientes com drenagem para o sistema venoso profundo, evidenciada previamente pela angiografia. $\mathrm{Na}$ sequiência fazia-se a infusão lenta de 1 a $2 \mathrm{ml}$ de xilocaína a $1 \%$ sem vasoconstritor e, em seguida, 1 a $3 \mathrm{ml}$ de álcool absoluto, dependendo do tamanho da malformação, pela mesma via de acesso. Ao término, realizava-se enfaixamento levemente compressivo, o paciente permanecia em repouso por 10 a $15 \mathrm{mi}-$ nutos e então era liberado para retornar à sua residência. Todos os pacientes recebiam orientação para cuidados locais e analgesia simples, se necessário.

Foram realizadas múltiplas aplicações de álcool absoluto, num intervalo de 15 a 30 dias, com o objetivo de avaliar os resultados iniciais deste tipo de tratamento.

Tabela 1.

\begin{tabular}{|c|c|c|c|c|}
\hline Paciente & Localização das lesões & Sinais/sintomas & Tratamento prévio & Diagnóstico \\
\hline 1) 25 anos/Fem. & Mão e antebraço esquerdos & Tumoração, dor & Uma ressecção há nove anos & Clínica + angiografia \\
\hline 2) 29 anos/Fem. & Mão direita & Dor & - & Clínica + angiografia \\
\hline 3) 28 anos/Masc. & Nádega e virilha esquerdas & Dor & - & Clínica + angiografia \\
\hline 4) 18 anos/Fem. & Antebraço direito & Dor, tumoração & - & Clínica + angiografia \\
\hline 5) 34 anos/Fem. & Cotovelo esquerdo & Dor, tumoração & - & Clínica + angiografia \\
\hline 6) 65 anos/Fem. & Quinto quirodáctilo direito & Tumoração & Duas ressecções anteriores & Clínica + angiografia \\
\hline 7) 27 anos/Fem. & Hálux e antepé esquerdos & $\begin{array}{l}\text { Dor, tumoração, dificuldade } \\
\text { para deambular }\end{array}$ & - & Clínica + angiografia \\
\hline 8) 17 anos/Fem. & Dorso do pé esquerdo & Dor, tumoração & - & Clínica + angiografia \\
\hline 9) 22 anos/Masc. & Mão direita & Problema estético & - & Clínica + angiografia \\
\hline 10) 25 anos/Masc. & Região glútea esquerda & Dor, tumoração & - & Clínica + angiografia \\
\hline 11) 19 anos/Fem. & Coxa direita & Dor, tumoração & - & Clínica + angiografia \\
\hline 12) 24 anos/Fem. & $\begin{array}{l}\text { Cotovelo direito, face ântero- } \\
\text { lateral }\end{array}$ & $\begin{array}{l}\text { Dor, tumoração, limitação } \\
\text { dos movimentos }\end{array}$ & - & Clínica + angiografia \\
\hline
\end{tabular}

Fem., feminino; Masc., masculino. 


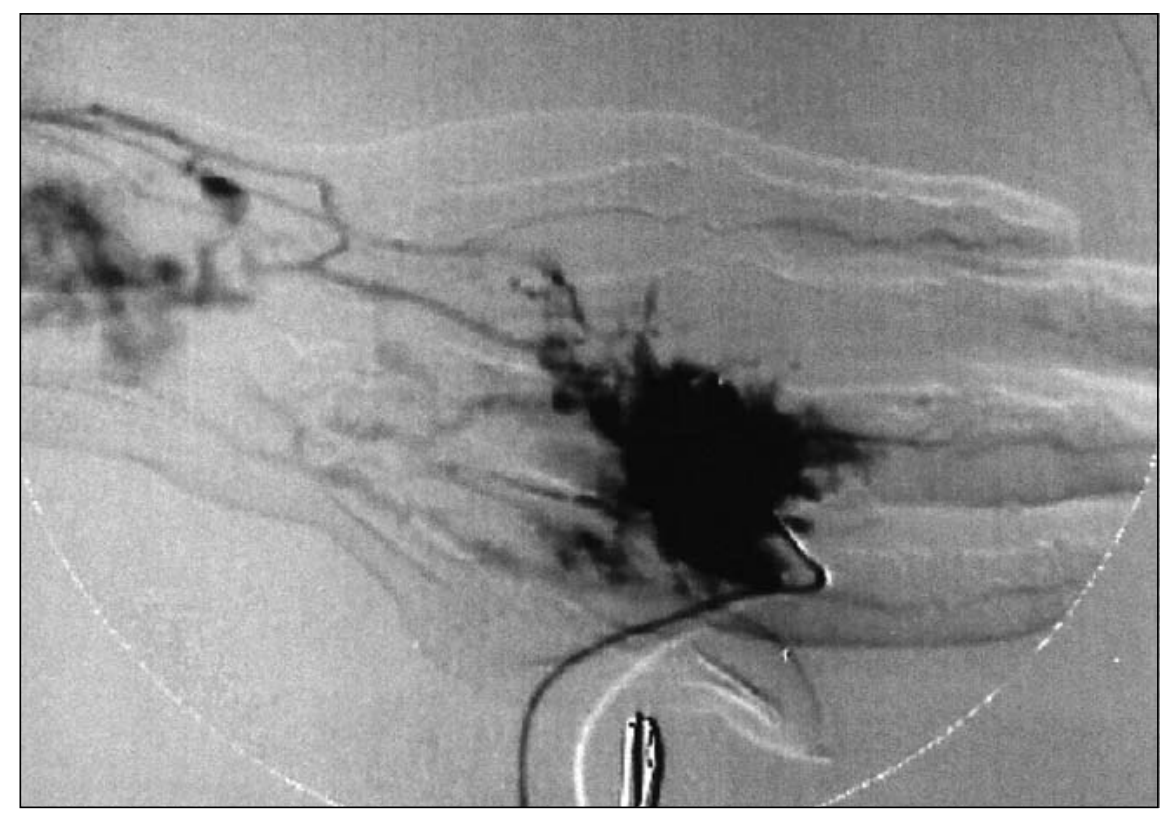

Figura 1. Angiografia venosa por punção direta pré-alcoolização demonstrando preenchimento da angiodisplasia e das veias de drenagem.

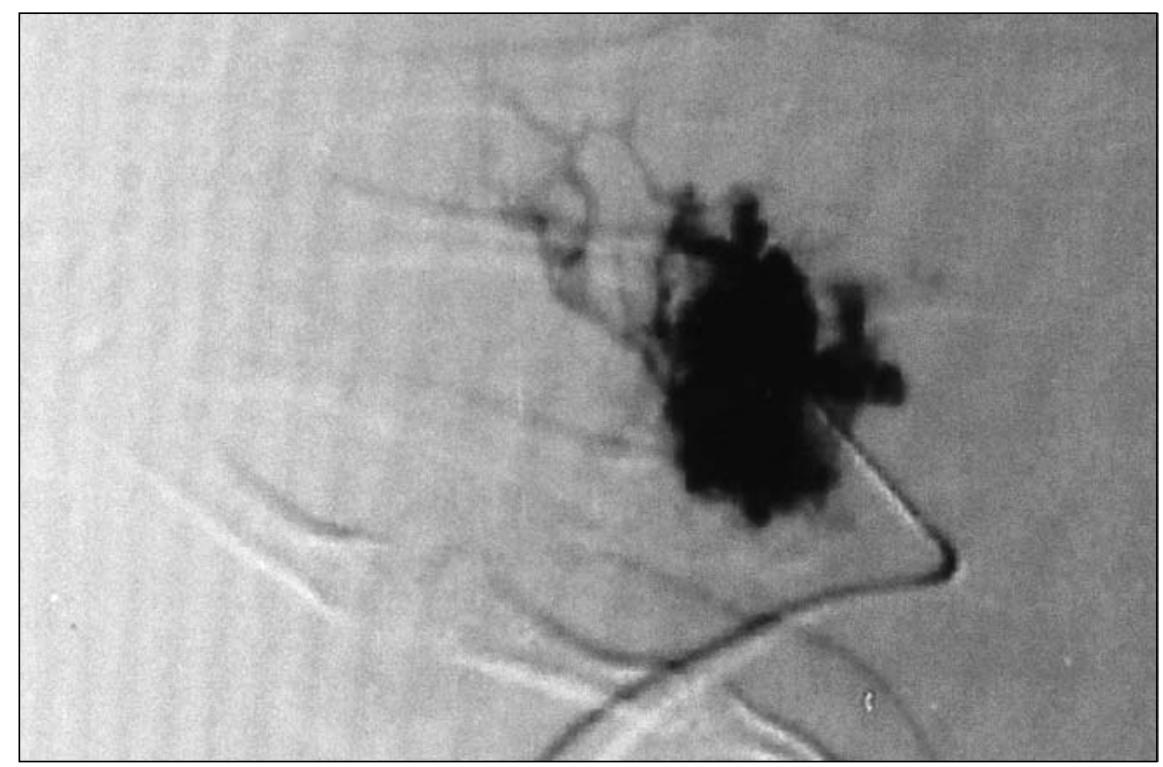

Figura 2. Controle angiográfico imediato após alcoolização com etanol mostrando diminuição do volume da angiodisplasia e das veias de drenagem.

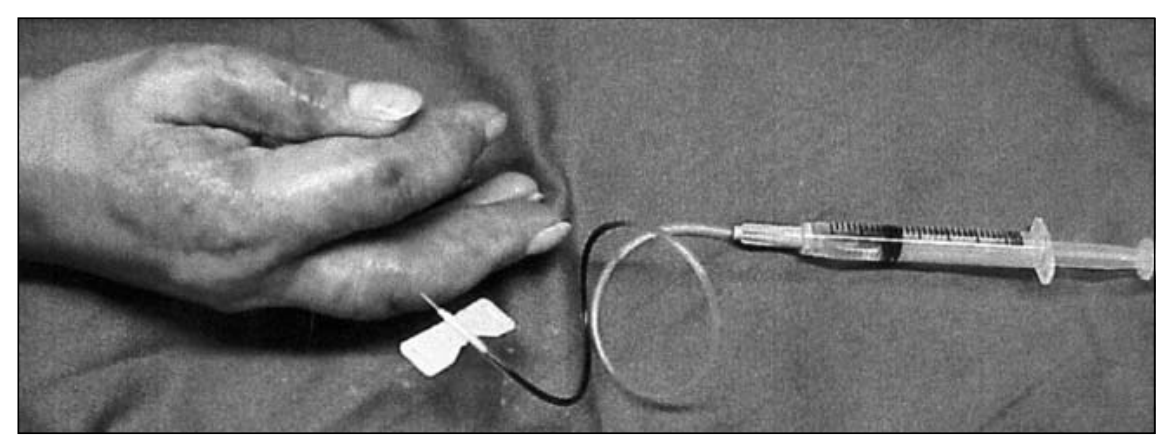

Figura 3. Demonstração da técnica utilizada para alcoolização percutânea por punção direta.

\section{RESULTADOS}

Doze pacientes foram submetidos a tratamento esclerosante com etanol, de suas malformações vasculares venosas, num total de 101 alcoolizações, variando de uma até 32 aplicações por paciente. Dez desses pacientes apresentaram melhora dos sintomas e diminuição do volume da lesão (Figuras 4 e 5).

O número de aplicações, tempo de seguimento, resultados, evolução e complicações são mostrados na Tabela 2 .

A quantidade de álcool absoluto utilizada em cada aplicação variou de 1 a 3 $\mathrm{ml}$, sempre com anestesia local, sendo bem tolerada na maioria das vezes. Alguns pacientes referiram gosto etílico na boca, logo após o procedimento.

O seguimento destes pacientes foi de duas semanas a 23 meses.

As complicações imediatas mais relatadas foram ardência durante as aplicações (100\% dos pacientes), cessando em 24 horas, e reação inflamatória local discreta, apresentando alívio com analgésicos comuns em todos os casos. Hipercromia no local da punção também foi achado comum (Figura 6).

Seis pacientes suspenderam o tratamento em virtude de melhora importante dos sinais e sintomas. Um deles interrompeu o tratamento após a segunda aplicação, por apresentar complicações como hiperemia do membro superior e linfonodomegalia dolorosa axilar. Outro suspendeu, por intolerância à dor durante a aplicação. Quatro pacientes estão em tratamento, já com melhora significativa dos sintomas e diminuição do volume da anomalia venosa.

Pequenas úlceras de pele no local da alcoolização ocorreram em três pacientes, não ultrapassando $2 \mathrm{~cm}$ de diâmetro, tendo em todos os casos resolução espontânea somente com cuidados locais (Figura 7).

Não se observaram recidivas ou agravamento dos sintomas no decorrer do período de acompanhamento.

\section{DISCUSSÃO}

As malformações vasculares constituem um desafio no acompanhamento 


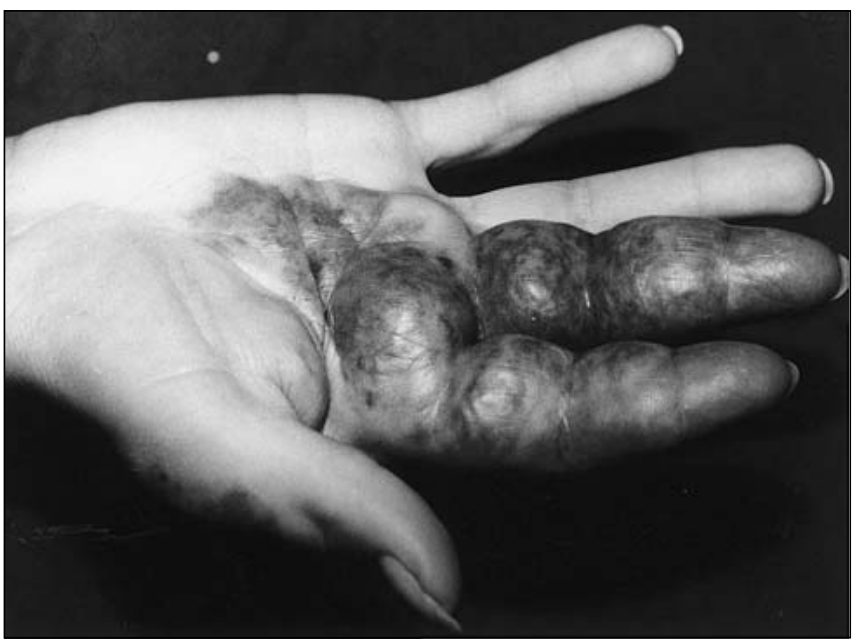

Figura 4. Malformação vascular venosa na mão, pré-alcoolização.

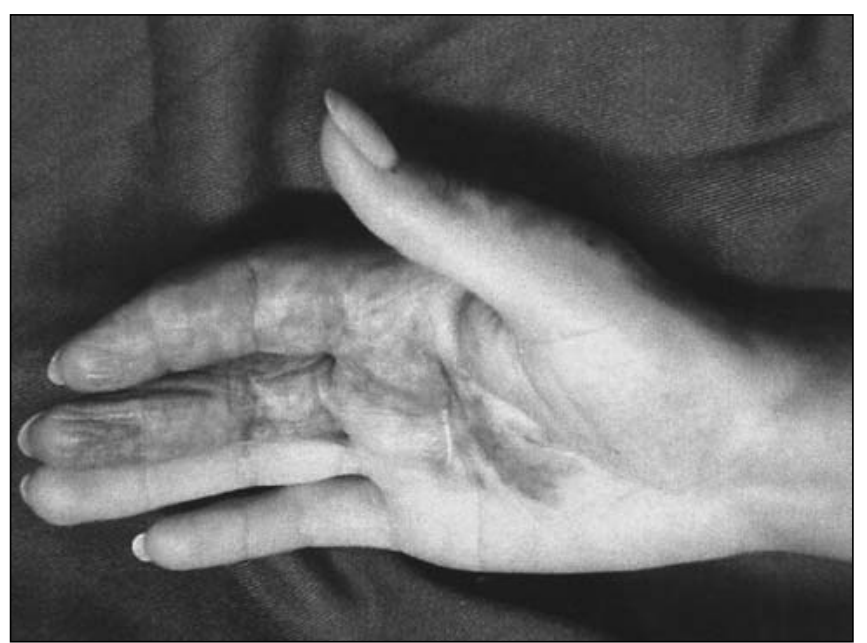

Figura 5. Foto de controle após 16 aplicações de álcool absoluto, com bom resultado.

Tabela 2.

\begin{tabular}{|l|c|l|l|l|l|}
\hline Paciente & Aplicações & Seguimento & Resultados & Evolução & Complicação \\
\hline 1) 25 anos/Fem. & 27 & 23 meses & Melhora & Continua & Três úlceras \\
2) 29 anos/Fem. & 32 & 26 meses & Melhora & Continua & Uma úlcera \\
3) 28 anos/Masc. & 16 & 21 meses & Melhora & Continua & Não \\
4) 18 anos/Fem. & 3 & Dois meses & Melhora & Terminou & Não \\
5) 34 anos/Fem. & 2 & Dois meses & Não-avaliado & Suspenso & Linfonodomegalia \\
6) 65 anos/Fem. & 1 & Duas semanas & Melhora & Terminou & Não \\
7) 27 anos/Fem. & 3 & Dois meses & Melhora & Terminou & Não \\
8) 17 anos/Fem. & 1 & Duas semanas & Não-avaliado & Desistiu & Não \\
9) 22 anos/Masc. & 5 & Três meses & Melhora & Terminou & Uma úlcera \\
10) 25 anos/Masc. & 7 & Quatro meses & Melhora & Terminou & Não \\
11) 19 anos/Fem. & 1 & Um mês & Melhora & Terminou & Não \\
12) 24 anos/Fem. & 3 & Três meses & Melhora & Continua & Não \\
\hline
\end{tabular}

Fem., feminino; Masc., masculino.

clínico e no seu tratamento. Frequientemente, as chances de cura com cirurgia são poucas, e ressecções incompletas podem exacerbar os sintomas ou tornar o paciente assintomático ou sintomático durante o seguimento ${ }^{(4,14)}$.

História de tratamentos prévios e ressecções cirúrgicas sem sucesso é comum. Em nossa casuística, apenas dois pacientes relataram ressecções cirúrgicas anteriores.

A técnica de esclerose por meio de punção percutânea direta, anestesia local e infusão de pequeno volume de etanol apresenta as vantagens de ser um procedimento fácil, simples e rápido, de baixo custo, bem tolerado na maioria dos casos, sem necessidade de internação e com poucas complicações ${ }^{(4)}$.
Segundo Shireman et al. ${ }^{(18)}$, outra forma de conduzir estas malformações é infundindo maior quantidade de álcool absoluto. As desvantagens deste método são: necessidade de internação, realização de anestesia geral por causa de dor intensa, utilização de analgésicos potentes e corticóides após o efeito da anestesia geral; as chances de complicações como necrose tissular tornam-se maiores, em alguns casos necessitando reparação cirúrgica ou até enxerto de pele. A vantagem está na resolução mais rápida, ao contrário do nosso método, em que anomalias extensas necessitam de várias aplicações de álcool, tornando o tratamento prolongado.

O número de aplicações dependeu do volume, extensão da malformação e da resposta ao tratamento adotado, variando de uma até 32 injeções por paciente.

$\mathrm{O}$ álcool atua na parede do vaso causando desidratação e desnaturação protéica, com ativação da cascata de coagulação, levando a trombose e organização fibrótica importante, com oclusão imediata e permanente do vaso ${ }^{(\mathbf{1 , 1 7})}$.

As complicações que podem ocorrer com este tipo de tratamento são: tromboflebite, trombose venosa profunda, úlcera de pele, que pode evoluir para necrose extensa, distúrbios neurológicos como parestesias, que normalmente regridem espontaneamente ${ }^{(\mathbf{4 , 2 1})}$.

Observou-se formação de úlceras de pele menores que $2 \mathrm{~cm}$ em três pacientes, que ocorreu pelo extravasamento do etanol para o subcutâneo. A resolução 


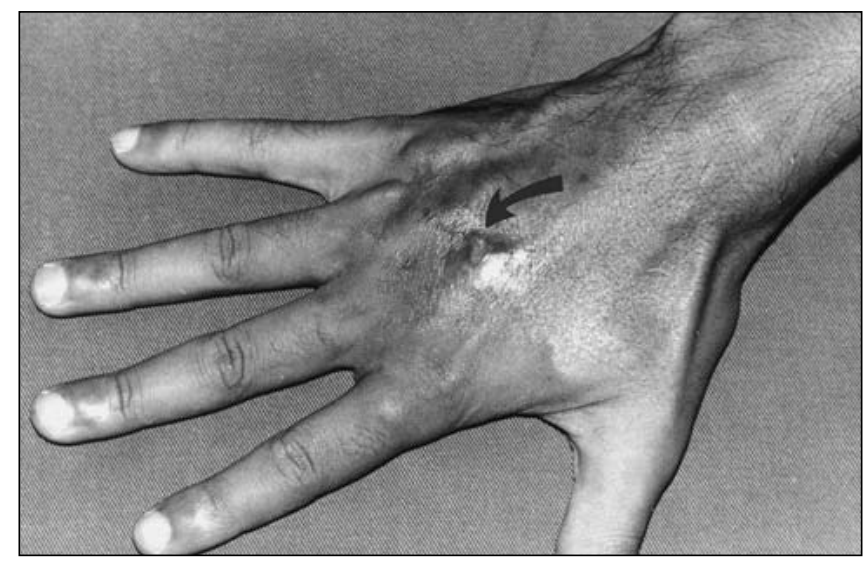

Figura 6. Hipercromia da pele na coxa após resolução da malformação com etanol (seta).

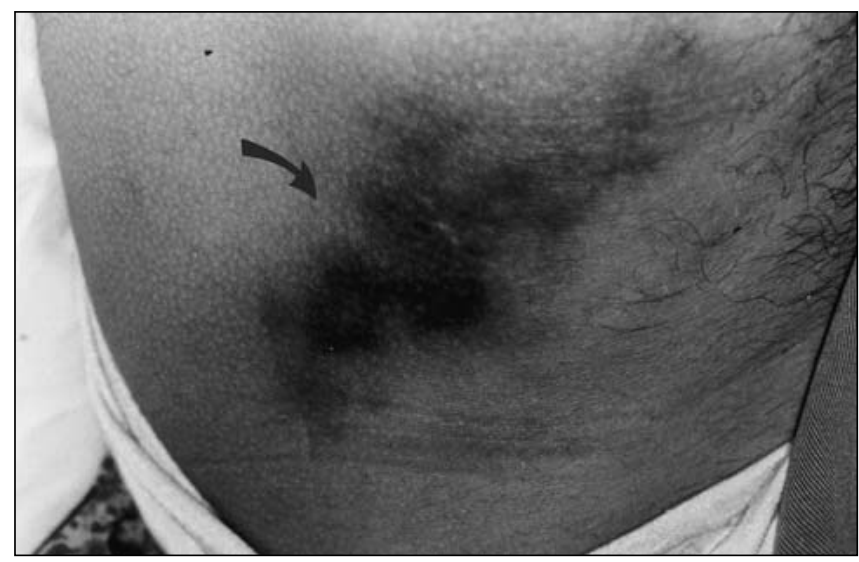

Figura 7. Formação de úlcera pós-tratamento percutâneo com álcoo (seta). espontânea com cuidados locais ocorreu em todos os casos, num prazo de duas a quatro semanas.

Não houve complicações sistêmicas no seguimento dos casos relatados. $\mathrm{Na}$ literatura são descritos casos de elevação dos níveis de álcool no sangue, sem repercussões clínicas, e aumento das taxas sanguíneas de creatino-fosfoquinase após alcoolização de malformações vasculares venosas extensas de membros inferiores, conseqüentes à grande destruição celular causada pelo álcool ${ }^{(3,20)}$.

\section{CONCLUSÃO}

A alcoolização percutânea utilizada de forma ambulatorial é importante opção terapêutica para anomalias vasculares venosas, muitas vezes irressecáveis cirurgicamente, apresentando bons resultados. Na maior parte dos casos, as complicações são discretas e a técnica é bem tolerada pelos pacientes, sendo os efeitos sistêmicos praticamente nulos.

\section{REFERÊNCIAS}

1. Gorriz Gómez E, Carreira Villamor JM, Reyes Pérez R, et al. Tratamiento percutáneo de las malformaciones vasculares periféricas. Rev Clín
Esp 1998;198:565-70

2. Mulliken JB, Glowacki J. Hemangiomas and vascular malformations in infants and children: a classification based on endothelial characteristics. Plast Reconstr Surg 1982;69:412-20.

3. Svendsen P, Wikholm G, Fogdestam I, Naredi S, Edén E. Instillation of alcohol into venous malformations of the head and neck. Scand J Plast Reconstr Hand Surg 1994;28:279-84.

4. Yakes WF, Haas DK, Parker SH, et al. Symptomatic vascular malformations: ethanol embolotherapy. Radiology 1989;170(3 Pt 2):1059-66.

5. Anger J. Os hemangiomas. Diagn Trat 1998;3:58 9 .

6. Burrows PE, Mulliken JB, Fellows KE, Strand RD. Childhood hemangiomas and vascular malformations: angiographic differentiation. AJR 1983;141:483-8.

7. Jackson IT, Carreno R, Potparic Z, Hussain K. Hemangiomas, vascular malformations, and lymphovenous malformations: classification and methods of treatment. Plast Reconstr Surg 1993;91: 1216-30.

8. Berthelsen B, Fogdestam I, Svendsen P. Venous malformations in the face and neck: radiologic diagnosis and treatment with absolute ethanol. Acta Radiol Diagn (Stockh) 1986;27:149-55.

9. Rosen RJ, Riles TS, Berenstein A. Congenital vascular malformations. In: Rutherford RB, ed. Vascular surgery. Philadelphia: Saunders, 1989: 1049.

10. Buetow PC, Kransdorf MJ, Moser RP Jr, Jelinek JS, Berrey BH. Radiologic appearance of intramuscular hemangioma with emphasis on MR imaging. AJR 1990;154:563-7.

11. Dubois J, Patriquin HB, Garel L, et al. Softtissue hemangiomas in infants and children: diagnosis using Doppler sonography. AJR 1998; 171:247-52.
12. Rak KM, Yakes WF, Ray RL, et al. MR imaging of symptomatic peripheral vascular malformations. AJR 1992;159:107-12.

13. Yakes WF, Pevsner P, Reed M, Donohue HJ, Ghaed N. Serial embolizations of an extremity arteriovenous malformation with alcohol via direct percutaneous puncture. AJR 1986;146:1038-40.

14. Erdmann MW, Jackson JE, Davies DM, Allison DJ. Multidisciplinary approach to the management of head and neck arteriovenous malformations. Ann R Coll Surg Engl 1995;77:53-9.

15. Górriz E, Carreira JM, Reyes R, et al. Intramuscular low flow vascular malformations: treatment by means of direct percutaneous embolization. Eur J Radiol 1998;27:161-5.

16. Mendel T, Louis DS. Major vascular malformations of the upper extremity: long-term observation. J Hand Surg 1997;22:302-6.

17. Muto T, Kinehara M, Takahara M, Sato K. Therapeutic embolization of oral hemangiomas with absolute ethanol. J Oral Maxillofac Surg 1990;48: 85-8.

18. Shireman PK, McCarthy WJ, Yao JST, Vogelzang $\mathrm{RL}$. Treatment of venous malformations by direct injection with ethanol. J Vasc Surg 1997;26:83844.

19. Takebayashi S, Hosaka M, Ishizuka E, Hirokawa M, Matsui K. Arteriovenous malformations of the kidneys: ablation with alcohol. AJR 1988;150: 587-90.

20. Vinson AM, Rohrer DG, Willcox CW, et al. Absolute ethanol embolization for peripheral arteriovenous malformation: report of two cures. South Med J 1988;81:1052-5.

21. Riche MC, Hadjean E, Tran-Ba-Huy P, Merland JJ. The treatment of capillary-venous malformations using a new fibrosing agent. Plast Reconstr Surg 1983;71:607-14. 